\title{
SOME NOTES ON THE AVERAGE DURATION OF AN INCOME PROTECTION CLAIM*
}

\author{
BY \\ Isabel Maria Ferraz Cordeiro
}

\begin{abstract}
Cordeiro (2002a) has presented a multiple state model for Income Protection (formerly known as Permanent Health Insurance) which enables us to analyse claims by cause of disability. In that paper average claim durations conditioned on recovery have been calculated.

Since, when an Income Protection claim is reported, the insurance company never knows whether it will end in recovery, in death or in expiry, to analyse only average claim durations conditioned on recovery could be misleading, specially for the people responsible for the claims control process.

The main purpose of this paper is to calculate average claim durations conditioned on death and average claim durations not conditioned on any particular mode of claim termination. We calculate these claim durations for different deferred periods, causes of disability and ages at the beginning of sickness and we analyse the results obtained.
\end{abstract}

\section{KEYWORDS}

Income Protection; Permanent Health Insurance; Multiple State Models; Analysis by Cause of Disability; Average duration of a claim; Average duration of a claim conditioned on death.

\section{Presentation of the Problem}

Income Protection (IP for brevity), formerly known as Permanent Health Insurance (see Continuous Mortality Investigation Committee (2001) for the reasons of the re-naming), is a class of long-term and non-cancellable sickness insurance which provides cover against the risk of loss of income due to disability. In general terms, an IP policy entitles the policyholder to an income during periods of disability longer than the deferred period specified in the policy. Benefits only start to be paid after the end of the deferred period.

* This research was supported by FCT - Fundação para a Ciência e Tecnologia, Portugal, under program POCTI. 
There are several types of IP policy sold by UK insurance companies. However, in this paper we are only interested in individual conventional policies with level benefits. This type of policy entitles the policyholder to a regular level income during periods of disability longer than the deferred period. In exchange for these benefits the policyholder has to pay a regular level premium throughout the term of the policy: from the time when he effects it, at which he is required to be healthy, to his 60th or 65th birthday (usually, the age of his retirement). In general, the premiums are waived whenever the policyholder is claiming.

We will assume throughout this paper that a policy expires when the policyholder reaches age 65 or dies, whichever occurs first. We will also assume that each policy has one of the following deferred periods: 1 week, 4 weeks, 13 weeks or 26 weeks (for brevity, throughout the paper we will refer to these deferred periods as D1, D4, D13 and D26, respectively).

Cordeiro (2002a) has presented a new multiple state model for IP which enables us to analyse claims by cause of disability. This model, which is a generalization of the model for IP proposed in Continuous Mortality Investigation Committee (1991), is very useful in the underwriting and claims control stages of IP business since it allows the calculation of quantities such as the average duration of a claim and claim inception rates by cause of disability.

In particular, as it is argued in Cordeiro (2002a), tables showing the average duration of a claim for the different classes of causes of disability, for the different deferred periods and for different ages at the beginning of sickness can help the underwriters in their decisions concerning proposals for new entries and the persons responsible for the claims control process in keeping a tighter control over the claims which are being paid and, therefore, in reducing claim recovery time.

In that paper, for illustrative purposes, examples of the tables mentioned above were shown. However, all the average claim durations in these tables were conditioned on recovery, i.e. they were all durations of claims which ended in recovery. It could be argued that, since for almost all the causes of disability the vast majority of claims ends in recovery, as we will see in Section 2, the important average claim durations for insurance companies would be those conditioned on recovery. However, to analyse only these average claim durations could be misleading, specially for the people responsible for the claims control process, since when a claim is reported, the insurance company never knows whether it will end in recovery, in death or in expiry (i.e. the sickness will not end before the corresponding policy expires). On the other hand, insurance companies have to take into consideration that, in general, as we will see in Section 2, the percentage of claims which end in death and in expiry increases with the length of the deferred period (becoming important for the longer deferred periods) and, for cause of disability malignant neoplasms, there is a great number of claims which end in death.

In view of the points made above, we think it is important that insurance companies should be able to analyse, not only average claim durations conditioned 
on recovery, but also average claim durations not conditioned on any particular mode of claim termination and conditioned on death. The purposes of this paper are to calculate the latter two average claim durations for different deferred periods, classes of causes of disability and ages at the beginning of sickness and analyse these claim durations. For those purposes, we use the approximations to the transition intensities defined for the model mentioned above obtained in Cordeiro (2002b).

The idea that insurance companies should analyse average durations not conditioned on any particular mode of claim termination is already implicit in the morbidity tables for valuation which the United States disability income industry has used for years. In fact, the termination rates given in the well known 85 CIDA tables do not differentiate terminations between recoveries and deaths (see Committee of the Society of Actuaries to Recommend New Disability Tables for Valuation (1985)). The same will happen in the new tables for valuation which are being created for the United States industry (see Individual Disability Experience Committee (2005)).

However, we should make two remarks on this matter. Firstly, both the studies which lead to the tables mentioned above are made in a different perspective, when compared to this paper. Their main purpose is to construct tables for calculating reserves both for healthy and sick policyholders (in the latter case, claim reserves). Our objective is to calculate average claim durations by cause of disability in order to help underwriters and, specially, the people responsible for the claims control process. On the other hand, in both tables mentioned above only recoveries and deaths (not expiries) are considered terminations. Furthermore, in the studies associated with these tables the impact of expiries on claim costs is not investigated.

In this paper we demonstrate that insurance companies should not analyse only average claim durations conditioned on recovery by showing that deaths and expiries contribute to make claims not conditioned on any mode of termination, in general, much longer than those which end in recovery. We also show that, in a significant number of cases (i.e. combinations of deferred period, class of causes of disability and age at the beginning of sickness), expiries have a strong impact on average durations not conditioned on any mode of claim termination.

This paper is related directly to the works presented in Cordeiro (2002a, 2002b) and in Continuous Mortality Investigation Committee (1991) (for brevity we will refer to this report as CMIC (1991) throughout the paper). Other important references on the subject "multiple state models applied to life and disability insurance" are: Haberman and Pitacco (1999), Hoem (1969, 1972, 1976, 1988), Waters $(1984,1990)$ and Wolthuis (1994).

In Section 2, we describe briefly the model proposed in Cordeiro (2002a), we present the approximations to some of the transition intensities defined for this model and, finally, we give formulae for the average duration of a claim conditioned on death and the average duration of a claim not conditioned on any particular mode of claim termination. In Section 3, we present tables with 
claim durations conditioned on death and claim durations not conditioned on any mode of claim termination. We also analyse these results.

\section{Formulae for Average Claim Durations}

Firstly, let us describe briefly the model mentioned in Section 1, which is going to be the basis for our calculations.

This model has $(n+2)$ states $(n>1)$ : healthy (denoted by $H)$, dead (denoted by $D$ ), sick with a sickness from class 1 (denoted by $S_{1}$ ), sick with a sickness from class 2 (denoted by $S_{2}$ ), ..., sick with a sickness from class n (denoted by $S_{n}$ ). Each state $S_{i}$ represents a different class of causes of disability. These $n$ states, considered together, group all possible causes of disability.

The important quantities for this model are the transition intensities: $\sigma(i)_{x}$ (associated with the transitions from $H$ to $S_{i}$ ), $\rho(i)_{x, z}$ (associated with the transitions from $S_{i}$ to $\left.H\right), v(i)_{x, z}$ (associated with the transition from $S_{i}$ to $\left.D\right)(i=1$, $2, \ldots, n)$ and $\mu_{x}$ (associated with the transition from $H$ to $D$ ). The action of these transition intensities governs the movements of a policyholder between the $(n+2)$ states.

The transition intensities $\sigma(i)_{x}$ (for a fixed $i$ ) and $\mu_{x}$, which can be designated as sickness intensity for class $i$ and mortality of the healthy intensity, respectively, depend only on $x$, the policyholder's attained age. $\rho(i)_{x, z}$ and $v(i)_{x, z}$ (for a fixed $i$ ), which can be designated as recovery intensity and mortality of the sick intensity for class $i$, respectively, depend on $x$ and on $z$, the duration of the policyholder's current sickness. Obviously, the $\sigma(i)_{x}, \rho(i)_{x, z}$ and $v(i)_{x, z}$ depend not only on $x$, or on $x$ and $z$, but also on the class of causes of disability $i$ they are associated with.

Note that in the model we are considering there are no transitions between the states $S_{1}, S_{2}, \ldots, S_{n}$. Once the policyholder is in state $S_{i}$, he can enter state $S_{j}(i \neq j ; i, j=1,2, \ldots, n)$ only after transferring back to state $H$. In other words, in this model it is assumed that once the policyholder is suffering from a given condition, he can become sick with a different condition only after recovering from the first one.

At first sight, we could think that it would be more realistic to define for the model transitions between the states $S_{1}, S_{2}, \ldots, S_{n}$, but this is not the case. In fact, it is not uncommon for someone who is sick with a given illness to start suffering also from another condition or to recover from the original illness and within a very short period of time to become sick again but with a different illness. However, it is common practice for the insurance companies, even in the cases where a sickness stops and another starts within a very short period of time, to continue with the payments of benefits to the policyholder under the original claim and not to consider that the original claim has ended and a new one has started. Thus, not to allow these transitions in the model, not only is convenient, since it makes the model less complicated in mathematical terms, but also it is more consistent with the IP claims administration system and, ultimately, with the data available concerning IP claims. 
A full description and all the important mathematical and statistical aspects of the model mentioned above can be found in Cordeiro (2002a, 2002b).

In order to obtain approximations to the transition intensities mentioned above (excluding $\mu_{x}$ ), Cordeiro (2002b) has used the graduations of the transition intensities defined for the model proposed in CMIC (1991), already mentioned in Section 1, and a set of IP data from UK insurance companies produced by the Continuous Mortality Investigation Bureau of the Institute of Actuaries and the Faculty of Actuaries: the Cause of Disability Experience, Individual Standard Experience, 1979-82.

We should note that the model presented in CMIC (1991) has only three states: healthy, sick (which groups all possible causes of disability) and dead, and the transition intensities defined for the model are $\sigma_{x}, \rho_{x, z}, v_{x, z}$ and $\mu_{x}$ (the sickness intensity, the recovery intensity, the mortality of the sick intensity and the mortality of the healthy intensity, respectively). Graduations of these intensities have been obtained also in CMIC (1991).

We should also note that in the set of data mentioned above the claims are classified into the 18 sickness categories which are presented in Table 1. Taking into consideration this fact, Cordeiro (2002b) has assumed that the number of classes of causes of disability in our model is $n=18$ and obtained approximations to $\rho(i)_{x, z}, v(i)_{x, z}$ and $\sigma(i)_{x}$ for each of the 4 deferred periods we consider and each of these 18 sickness categories.

TABLE 1

SiCKNESS CATEGORIES INTO WHICH ARE CLASSIFIED THE DATA USED IN CORDEIRO (2002b) AND ESTIMATES OF THE CORRESPONDING $k_{i}$.

\begin{tabular}{rlr}
\hline \hline Sickness Category & $\widehat{k_{i}}$ \\
\hline 1 & Other Infective & 1.45 \\
2 & Malignant Neoplasms & 0.01 \\
3 & Benign Neoplasms & 1.45 \\
4 & Endocrine and Metabolic & 0.4 \\
5 & Mental Illness & 0.35 \\
6 & Nervous Disease & 0.9 \\
7 & Heart/Circulating System & 0.85 \\
8 & Ischaemic Heart Disease & 0.4 \\
9 & Cerebro Vascular Disease & 0.65 \\
10 & Acute Respiratory & 2.25 \\
11 & Bronchitis Respiratory & 1.65 \\
12 & Digestive & 1.2 \\
13 & Genito-Urinary & 1.35 \\
14 & Arthritis/Spondylitis & 0.5 \\
15 & Other Musculoskeletal & 1 \\
16 & R.T.A. Injuries & 0.8 \\
17 & Other Injuries & 1.05 \\
18 & All Others & 1 \\
\hline \hline
\end{tabular}


For a given deferred period, the approximations to the transition intensities have been obtained assuming that

$$
\begin{array}{llrl}
\rho(i)_{x, z} & =k_{i} \rho_{x, z} & i & =1, \ldots, 18 \\
v(i)_{x, z} & =w_{i} v_{x, z} & i & i, \ldots, 18 \\
\sigma(i)_{x} & =\exp \left\{\alpha_{i}+\beta_{i} x\right\} \sigma_{x} & i & i=1, \ldots, 18
\end{array}
$$

where $k_{i}, w_{i}, \alpha_{i}$ and $\beta_{i}(i=1, \ldots, 18)$ are unknown parameters which can be estimated from the data.

As far as the recovery and mortality of the sick intensities are concerned, this means it is assumed that, for a given deferred period, although $\rho(i)_{x, z}$ and $v(i)_{x, z}$ have the same shapes as $\rho_{x, z}$ and $v_{x, z}$, respectively, they can have different levels. In Table 1, for each of the 18 sickness categories we consider, we present the estimated level of the corresponding $\rho(i)_{x, z}$ (due to another assumption made by Cordeiro (2002b), this level is the same for the 4 deferred periods we consider). The approximation proposed for each $v(i)_{x, z}$ is the graduation of $v_{x, z}$ obtained in CMIC (1991) (which is the same for the 4 deferred periods we consider), except for sickness category 2 (malignant neoplasms), in which case the estimated level of $v(2)_{x, z}$ is much higher $\left(\widehat{w}_{2}=13.55\right.$, for the 4 deferred periods we consider), as we would expect.

Since we will not need to use the approximations to the $\sigma(i)_{x}$ and $\mu_{x}$ in this paper, we do not present here more information about them. A full description of the estimation processes which lead to the approximations to the transition intensities defined for our model can be found in Cordeiro (2002b).

It is important to note that, since the approximations to the $\rho(i)_{x, z}$ and $v(i)_{x, z}$ we use in this paper have been obtained with data from 1979-82, it is very likely that the average claim durations we present in Section 3 are not updated. In fact, we have to consider that changes over time in different factors, such as medical technology and claims management techniques, imply changes in the transition intensities. The ideal situation would be to work with graduations of the intensities based on more recent data but, unfortunately, these graduations are not available.

The fact that the transition intensities have been changing with time is confirmed by the investigations carried out in successive Continuous Mortality Investigation reports (CMIC (1996), CMIC (2000), CMIC (2001) and CMIC (2005)). These investigations try to identify trends in IP claims data for successive quadrennia by using a methodology based on a comparison of actual versus expected recoveries and deaths (analysed separately). This methodology was proposed initially in CMIC (1996). Unfortunately, the IP data analysed in the mentioned investigations are not by cause of disability and the data after 1990 refer to a wider IP experience (i.e. the experience to which our data refer is a subset of the experience analysed in those investigations).

More recently, an analysis of IP data by cause of disability covering the period 1975-2002 has become available (see Income Protection Committee (2006)). One 
of the purposes of this work is also to analyse trends in the data. However, neither these data are classified into exactly the same sickness categories as our data, nor a statistically robust analysis of these data (e.g. an analysis based on the methodology proposed in CMIC (1996)) has been carried out (only a simple statistical data analysis has been presented). Furthermore, the data after 1990 also refer to the wider IP experience mentioned above.

Although it is not possible to use the information presented in Income Protection Committee (2006) in order to update the approximations to our transition intensities, this information suggests it is very likely that many of the conclusions in Section 3 which result from comparisons between sickness categories are still valid today. This is certainly true for many of the conclusions concerning claims which end in recovery presented in Cordeiro (2002a), where the same approximations are used (e.g. the sickness categories which have the shorter and the longer claim durations are broadly the same; it is still true that, in general, the average duration of a claim increases as the deferred period becomes longer and that the $v(2)_{x, z}$ dominate the corresponding intensities for all the other sickness categories, making the category malignant neoplasms the main responsible for deaths).

Table 2 shows a summary of part of the set of IP data mentioned above. A full account of the characteristics and limitations of these data can be found in Cordeiro (2002b).

TABLE 2

NUMBER OF CLAIMS WHICH ENDED IN RECOVERY, IN DEATH AND IN EXPIRY.

\begin{tabular}{|c|c|c|c|c|c|c|c|c|c|c|c|c|}
\hline \multirow{2}{*}{$\begin{array}{l}\text { Sick. } \\
\text { Cat. }\end{array}$} & \multicolumn{4}{|c|}{$\begin{array}{c}\text { Number of claims which } \\
\text { ended in Recovery }\end{array}$} & \multicolumn{4}{|c|}{$\begin{array}{c}\text { Number of claims which } \\
\text { ended in Death }\end{array}$} & \multicolumn{4}{|c|}{$\begin{array}{c}\text { Number of claims which } \\
\text { ended in Expiry }\end{array}$} \\
\hline & D1 & D4 & D13 & D26 & D1 & D4 & D13 & D26 & D1 & D4 & D13 & D26 \\
\hline 2 & 67 & 27 & 13 & 8 & 25 & 20 & 36 & 19 & 2 & 1 & 2 & 5 \\
\hline All S.C. & 5423 & 1255 & 525 & 164 & 73 & 59 & 72 & 59 & 77 & 45 & 69 & 87 \\
\hline
\end{tabular}

As we can see from Table 2, the percentage of claims which ended in death and in expiry increases with the length of the deferred period, being $47.1 \%$ for deferred period D26. We can also see that, for sickness category 2 (malignant neoplasms), the number of claims which ended in death is significant for all the deferred periods considered (for D13, it represents half of the claims which ended in death for all causes of disability).

Furthermore, if we analyse the set of data mentioned above, we can find a significant number of cases where, although the number of claims which ended in death or in expiry is small, the average duration of these claims is extremely long (these data are not shown). There are even a few cases where the number of claims which ended in expiry is significant and the corresponding average duration is quite long. 
Let us give some examples of such cases. For sickness category 8 (ischaemic heart disease) and deferred periods D1 and D26, there are 22 and 17 claims which ended in expiry, respectively, and the corresponding average durations are 207.8 and 199.3 weeks, respectively. For sickness category 5 (mental illness) and D13, the number of claims which ended in death is 4 and the respective average duration is 384.5 weeks. Also for sickness category 5 and D26, the number of claims which ended in expiry is 12 and the corresponding average duration is 413 weeks.

Considering all the facts mentioned above, as we have stated in Section 1, insurance companies selling IP policies (specially the people responsible for the claims control process) can only benefit from analysing, not only average claim durations conditioned on recovery, but also average claim durations not conditioned on any mode of claim termination and conditioned on death. We should note that, for a given age at the beginning of sickness, the duration of a claim which ends in expiry is obvious.

Cordeiro (2002a) has presented the formula for the average duration of a claim conditioned on recovery for a given class of causes of disability. If we denote by $T_{i y}^{r}$ the duration of a claim in class $i$, which ends in recovery, for a claimant aged $y$ at the beginning of the corresponding sickness, the average duration of this claim (in years) is given by

$$
E\left(T_{i y}^{r}\right)=\frac{\int_{d}^{65-y}{ }_{t} p_{y} \overline{S_{i} S_{i}} \rho(i)_{y+t, t} d t}{\int_{d}^{65-y}{ }_{t}{ }_{t} \overline{S_{i} S_{i}} \rho(i)_{y+t, t} d t}-d \quad i=1,2, \ldots, n
$$

where $d$ is the deferred period expressed in years and ${ }_{t} p_{y} \overline{S_{i} S_{i}}$ is the notation for ${ }_{t} \overline{S_{y+z, z} S_{i}}$ with $z=0$, being

$$
{ }_{t} p_{y+z, z}^{\overline{S_{i} S_{i}}}=\exp \left\{-\int_{0}^{t}\left(\rho(i)_{y+z+s, z+s}+v(i)_{y+z+s, z+s}\right) d s\right\}
$$

the probability of a policyholder remaining sick until at least age $(y+z+t)$, given that he is sick at age $(y+z)$ with a sickness from class $i$ and with duration of sickness $z \cdot{ }_{t} p_{y+z, z} \overline{S_{i} S_{i}}$ is a basic probability for our model and the derivation of its formula can be found in Cordeiro (2002a).

With two simple changes of variable and some trivial manipulation, it can be shown that formula (2) is equal to:

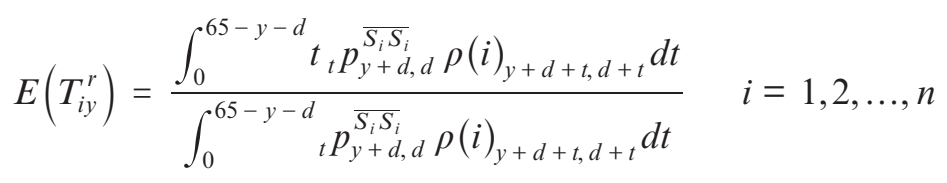

In order to obtain the formula for the average duration of a claim conditioned on death, we only have to make small changes in formula (4). In fact, if we 
denote by $T_{i y}^{m}$ the duration of a claim in class $i$, which ends in death, for a claimant aged $y$ at the beginning of the corresponding sickness, the average duration of this claim (in years) is given by

$$
E\left(T_{i y}^{m}\right)=\frac{\int_{0}^{65-y-d} t_{t} p_{y+d, d}^{\overline{S_{i} S_{i}}} v(i)_{y+d+t, d+t} d t}{\int_{0}^{65-y-d}{ }_{t} p_{y+d, d} \overline{S_{i} S_{i}} v(i)_{y+d+t, d+t} d t} \quad i=1,2, \ldots, n
$$

Since when a claim is reported, it is not known in advance whether it will end in recovery, in death or in expiry, it would be useful for the insurance company to have available tables with average claim durations not conditioned on any particular mode of claim termination (i.e. average durations of claims which end in recovery or in death or in expiry while the corresponding policies are in force). The formula for the average duration of a claim in class $i$, not conditioned on any mode of claim termination, for a claimant aged $y$ at the beginning of the corresponding sickness, is the following:

$$
\begin{aligned}
E\left(T_{i y}\right)= & \int_{0}^{65-y-d} t_{t} p_{y+d, d}^{\overline{S_{i} S_{i}}}\left(\rho(i)_{y+d+t, d+t}+v(i)_{y+d+t, d+t}\right) d t \\
& +(65-y-d)_{65-y-d} p_{y+d, d} \overline{S_{i} S_{i}} \quad i=1,2, \ldots, n
\end{aligned}
$$

where $T_{i y}$ denotes the duration of such a claim. The first term on the right hand side of (6) concerns the case where the claim ends in recovery or in death whereas the second term concerns the case where the claim ends in expiry (i.e. the corresponding sickness ends in recovery or in death after the policy expires at age 65). $T_{i y}$ has a mixed distribution with positive density from 0 to $(65-y-d)$ and a probability mass at $(65-y-d)$ (the duration of the claim when it ends in expiry).

\section{Analysis of the Average Claim Durations}

In this section we use the approximations to the $\rho(i)_{x, z}$ and $v(i)_{x, z}$ proposed in Cordeiro (2002b) to calculate the average duration of a claim conditioned on death and the average duration of a claim not conditioned on any mode of claim termination for the 4 deferred periods we consider, the 18 sickness categories presented in Table 1 and different ages at the beginning of sickness $(20,25,30,35,40,45,50,55$ and 60$)$. The integrals in formulae (5) and (6) have been calculated numerically, using the repeated trapezoidal rule with a step size equal to $1 / 156$ of a year, and we assumed there are exactly 52 weeks in a year.

We should note that, although here, due to limitations of space, we show only the results for deferred periods D1 and D26 and ages at the beginning of sickness 20,30,40, 50 and 60, our conclusions refer to all the results obtained. We should also note that, as it can be noticed, the average claim durations 
TABLE 3

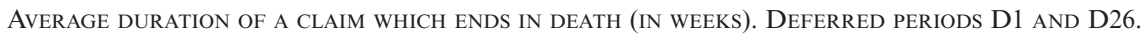

\begin{tabular}{l|r|r|r|r|r|r|r|r|r|r}
\hline \hline & \multicolumn{9}{c|}{ D1 } & \multicolumn{5}{c}{ D26 } \\
Sick. \\
Cat.
\end{tabular}

have the same values for some pairs of sickness categories ( 1 and 3; 4 and 8; 15 and 18). The reason for this is the fact that the sickness categories in each pair have the same approximations to $\rho(i)_{x, z}$ and $v(i)_{x, z}$ (see Section 2).

The average claim durations conditioned on death (in weeks) are shown in Table 3.

The most prominent feature in this table is that, in general, the average claim durations are very long. Furthermore, there is a significant number of cases where the claim durations are extremely long (see, for example, the claim durations for sickness categories 4 (endocrine and metabolic), 5 (mental illness), 8 (ischaemic heart disease) and 14 (arthritis/spondylitis), the younger ages at the beginning of sickness and all the deferred periods we consider).

If we compare these durations with the corresponding average claim durations conditioned on recovery, which appear in Table 4 (the values in this table are extracted from Cordeiro (2002a, Tables 3 to 6)), we can see that, for a given combination of deferred period, sickness category and age at the beginning of sickness, the average duration of a claim conditioned on death is always greater than the average duration of a claim conditioned on recovery. We were expecting this feature. In fact, if we consider the model described in Section 2 and 
TABLE 4

Average duration of a Claim Which ends in ReCovery (In WeEKS). Deferred Periods D1 AND D26.

\begin{tabular}{|c|c|c|c|c|c|c|c|c|c|c|}
\hline \multirow{3}{*}{$\begin{array}{l}\text { Sick. } \\
\text { Cat. }\end{array}$} & \multicolumn{5}{|c|}{ D1 } & \multicolumn{5}{|c|}{ D26 } \\
\hline & \multicolumn{5}{|c|}{ Age at the Beginning of Sickness } & \multicolumn{5}{|c|}{ Age at the Beginning of Sickness } \\
\hline & 20 & 30 & 40 & 50 & 60 & 20 & 30 & 40 & 50 & 60 \\
\hline 1 & 0.84 & 1.16 & 1.74 & 2.75 & 3.93 & 41.43 & 55.36 & 64.11 & 56.96 & 32.53 \\
\hline 2 & 14.47 & 12.76 & 10.68 & 8.68 & 7.02 & 53.47 & 44.61 & 35.71 & 27.06 & 17.62 \\
\hline 3 & 0.84 & 1.16 & 1.74 & 2.75 & 3.93 & 41.43 & 55.36 & 64.11 & 56.96 & 32.53 \\
\hline 4 & 20.28 & 21.65 & 20.11 & 15.66 & 10.20 & 198.49 & 170.69 & 129.73 & 81.35 & 36.50 \\
\hline 5 & 26.30 & 26.40 & 23.14 & 17.11 & 10.67 & 213.74 & 179.58 & 133.89 & 82.67 & 36.69 \\
\hline 6 & 2.32 & 3.49 & 5.08 & 6.39 & 6.42 & 92.02 & 100.25 & 93.45 & 68.89 & 34.57 \\
\hline 7 & 2.71 & 4.07 & 5.76 & 6.97 & 6.72 & 99.40 & 105.86 & 96.65 & 70.07 & 34.76 \\
\hline 8 & 20.28 & 21.65 & 20.11 & 15.66 & 10.20 & 198.49 & 170.69 & 129.73 & 81.35 & 36.50 \\
\hline 9 & 5.91 & 8.13 & 9.89 & 9.97 & 8.10 & 135.56 & 131.37 & 110.39 & 74.93 & 35.53 \\
\hline 10 & 0.44 & 0.58 & 0.81 & 1.27 & 2.19 & 17.49 & 25.85 & 37.37 & 42.86 & 29.71 \\
\hline 11 & 0.69 & 0.92 & 1.35 & 2.16 & 3.34 & 32.17 & 45.05 & 55.87 & 53.08 & 31.81 \\
\hline 12 & 1.19 & 1.71 & 2.62 & 3.91 & 4.88 & 58.65 & 72.31 & 76.16 & 62.15 & 33.45 \\
\hline 13 & 0.95 & 1.33 & 2.02 & 3.14 & 4.28 & 47.43 & 61.54 & 68.68 & 58.99 & 32.90 \\
\hline 14 & 12.13 & 14.54 & 15.13 & 13.09 & 9.31 & 170.72 & 153.93 & 121.71 & 78.74 & 36.11 \\
\hline 15 & 1.77 & 2.65 & 3.99 & 5.39 & 5.85 & 78.96 & 89.89 & 87.32 & 66.58 & 34.20 \\
\hline 16 & 3.22 & 4.78 & 6.56 & 7.61 & 7.04 & 107.41 & 111.77 & 99.94 & 71.26 & 34.95 \\
\hline 17 & 1.58 & 2.34 & 3.57 & 4.96 & 5.59 & 73.21 & 85.12 & 84.40 & 65.45 & 34.01 \\
\hline 18 & 1.77 & 2.65 & 3.99 & 5.39 & 5.85 & 78.96 & 89.89 & 87.32 & 66.58 & 34.20 \\
\hline
\end{tabular}

that, in general, for a given sickness category $i$, the graduated values of $\rho(i)_{x, z}$ are much higher than those of $v(i)_{x, z}$ (see Cordeiro (2002a, 2002b) and CMIC (1991)), it is easy to see that, on average, a policyholder remains more time in the state $S_{i}$ before he dies from a sickness in category $i$ than before he recovers from a sickness in the same category.

The fact that, in general, the claims which end in death are much longer than the claims which end in recovery confirms that underwriters and the people responsible for the claims control process should analyse not only claim durations conditioned on recovery but also claim durations conditioned on death. This conclusion is relevant specially when considering claims in sickness category 2 (malignant neoplasms), where there is a significant number of deaths, and claims in categories where the average claim durations conditioned on death are extremely long.

Another prominent feature in Table 3 is that, in general, for a given combination of sickness category and age at the beginning of sickness, the average duration of a claim increases as the deferred period becomes longer (this does not happen only when we compare the claim durations for D4, sickness category 2 and ages at the beginning of sickness higher than 40 with the corresponding ones 
for D1). We also observe this feature in the average claim durations conditioned on recovery (see Table 4 and Cordeiro (2002a)). The explanation for this feature is the same as for the claim durations conditioned on recovery: the average duration of a claim tends to be longer for longer deferred periods because in the calculation of the average duration, for a given deferred period $d$, we only consider sicknesses with durations longer than $d$ (see formula (5)).

We can also see from Table 3 that, for most of the combinations of deferred period and sickness category, the average duration of a claim conditioned on death increases with age at the beginning of sickness up to some age and then decreases. For all the deferred periods we consider, there are a few sickness categories for which the average duration of a claim always decreases with $y$ (the number of such cases tends to increase as the deferred period becomes longer). We also observe these features in the average claim durations conditioned on recovery (see Table 4 and Cordeiro (2002a)). However, in Table 3: in general, the claim durations start to decrease at earlier ages; for D1, we observe a greater number of cases where the average duration is a decreasing function of $y$; and, unlike in the average durations conditioned on recovery, we do not see any case where the average duration always increases with $y$.

While having an average duration which increases up to some age and then decreases can be considered as a non-intuitive feature for claims which end in recovery (see Cordeiro (2002a)), this is not the case for claims which end in death. In fact, we would expect the average duration of a claim conditioned on death to decrease with $y$, at least after some age.

The explanations for the average claim durations which decrease after some age (or which always decrease with $y$ ) are the same as those given for the claim durations conditioned on recovery: the behaviour of the probability ${ }_{t} p_{y+d, d} S_{i} S_{i}$ when $y$ increases, which depends on the levels and shapes of $\rho(i)_{x, z}$ and $v(i)_{x, z}$, and the policy expiration effect (i.e. claims have to be shorter than $(65-y-d)$ ) (see Cordeiro (2002a)). As far as the former explanation is concerned, note that the average claim durations which always decrease with $y$ are those for the sickness categories with the $\rho(i)_{x, z}$ having the lowest levels: sickness categories 2, 4, 5, 8, 9 and 14 (malignant neoplasms, endocrine and metabolic, mental illness, ischaemic heart disease, cerebro-vascular disease and arthritis/spondilytis, respectively; see Table 1).

A reason that can be given for claim durations conditioned on death starting to decrease at earlier ages than those conditioned on recovery (and also for not having durations conditioned on death which always increase with age) is that the longer the claim durations, the stronger is the policy expiration effect. As we have seen above, in general, the claim durations conditioned on death are very long.

The average claim durations not conditioned on any mode of claim termination (in weeks) are shown in Table 5.

We can see from Tables 3, 4 and 5 that, for a given combination of deferred period, sickness category and age at the beginning of sickness, the average duration of a claim not conditioned on any mode of claim termination is 
TABLE 5

AVERAGE DURATION OF A CLAIM NOT CONDITIONED ON ANY MODE OF CLAIM TERMINATION (IN WEEKS). DEFERRED PERIODS D1 AND D26.

\begin{tabular}{|c|c|c|c|c|c|c|c|c|c|c|}
\hline \multirow{3}{*}{$\begin{array}{l}\text { Sick. } \\
\text { Cat. }\end{array}$} & \multicolumn{5}{|c|}{ D1 } & \multicolumn{5}{|c|}{ D26 } \\
\hline & \multicolumn{5}{|c|}{ Age at the Beginning of Sickness } & \multicolumn{5}{|c|}{ Age at the Beginning of Sickness } \\
\hline & 20 & 30 & 40 & 50 & 60 & 20 & 30 & 40 & 50 & 60 \\
\hline 1 & 0.87 & 1.21 & 2.04 & 4.47 & 8.76 & 48.95 & 81.22 & 133.03 & 183.08 & 127.97 \\
\hline 2 & 76.46 & 67.06 & 53.21 & 39.59 & 28.13 & 113.25 & 96.16 & 75.25 & 55.39 & 37.24 \\
\hline 3 & 0.87 & 1.21 & 2.04 & 4.47 & 8.76 & 48.95 & 81.22 & 133.03 & 183.08 & 127.97 \\
\hline 4 & 55.31 & 81.71 & 109.90 & 123.64 & 79.94 & 478.13 & 517.08 & 505.01 & 407.47 & 174.70 \\
\hline 5 & 81.38 & 112.46 & 141.27 & 148.56 & 89.83 & 546.14 & 571.13 & 540.39 & 423.80 & 177.35 \\
\hline 6 & 2.73 & 5.15 & 10.81 & 21.40 & 26.01 & 141.41 & 201.22 & 261.48 & 276.60 & 150.46 \\
\hline 7 & 3.34 & 6.43 & 13.31 & 25.26 & 28.97 & 158.14 & 220.13 & 278.80 & 287.39 & 152.71 \\
\hline 8 & 55.31 & 81.71 & 109.90 & 123.64 & 79.94 & 478.13 & 517.08 & 505.01 & 407.47 & 174.70 \\
\hline 9 & 9.58 & 18.04 & 32.58 & 50.27 & 45.09 & 253.12 & 318.66 & 361.74 & 335.27 & 162.09 \\
\hline 10 & 0.46 & 0.60 & 0.84 & 1.36 & 2.83 & 18.05 & 29.50 & 56.09 & 103.63 & 101.60 \\
\hline 11 & 0.71 & 0.95 & 1.47 & 2.95 & 6.24 & 36.03 & 60.83 & 105.65 & 158.21 & 120.73 \\
\hline 12 & 1.24 & 1.92 & 3.74 & 8.50 & 14.01 & 76.22 & 120.32 & 179.58 & 220.43 & 137.70 \\
\hline 13 & 0.98 & 1.42 & 2.52 & 5.68 & 10.51 & 57.97 & 94.65 & 149.77 & 197.11 & 131.77 \\
\hline 14 & 26.30 & 43.75 & 66.96 & 85.91 & 63.44 & 368.52 & 424.91 & 441.47 & 376.78 & 169.53 \\
\hline 15 & 1.96 & 3.47 & 7.30 & 15.49 & 21.04 & 113.92 & 168.71 & 230.29 & 256.32 & 146.07 \\
\hline 16 & 4.19 & 8.15 & 16.50 & 29.90 & 32.31 & 177.26 & 241.08 & 297.38 & 298.63 & 155.00 \\
\hline 17 & 1.71 & 2.92 & 6.09 & 13.25 & 18.96 & 102.63 & 154.75 & 216.26 & 246.79 & 143.92 \\
\hline 18 & 1.96 & 3.47 & 7.30 & 15.49 & 21.04 & 113.92 & 168.71 & 230.29 & 256.32 & 146.07 \\
\hline
\end{tabular}

always greater than the average duration of a claim which ends in recovery and, for some cases, it is even greater than the average duration of a claim which ends in death. Furthermore, when we consider all the results obtained, the number of the latter cases seems to be an increasing function of simultaneously the age at the beginning of sickness, the inverse of the level of the approximation to $\rho(i)_{x, z}$ for sickness category $i\left(1 / \widehat{k_{i}}\right)$ and the length of the deferred period, becoming quite significant for deferred periods D13 (32 cases out of 162) and D26 (55 cases).

The fact that, on average, a claim not conditioned on any mode of claim termination is always longer than a claim which ends in recovery is not at all surprising. In fact, the claims not conditioned on any mode of claim termination comprise also the claims which end in death (which, on average, are longer than the claims which end in recovery) and the claims which end in expiry (which have the longest possible durations).

On the other hand, the other features mentioned in the paragraph before last seem to suggest that the claims which end in expiry are a phenomenon which can become important (in the sense that, on average, it can make the claims 
even longer than those which end in death), specially for the cases where the age at the beginning of sickness is high, the sickness category has a $\rho(i)_{x, z}$ with a low level and the deferred period is long.

In order to throw some light on the matter, we decided to calculate ${ }_{65-y-d} p_{y+d, d} S_{i} S_{i}$ (i.e. the probability that a claim in sickness category $i$, for a claimant aged $y$ at the beginning of sickness, ends in expiry) for all the deferred periods we consider and some sickness categories and ages at the beginning of sickness. Part of these results are shown in Table 6 (we do not show the results for deferred periods D4 and D13).

TABLE 6

Probability of a Claim ending In EXPIRY. Deferred Periods D1 AND D26.

\begin{tabular}{c|c|c|c|c|c|c|c|c}
\hline \hline \multirow{2}{*}{$\begin{array}{c}\text { Sickness } \\
\text { Category }\end{array}$} & \multicolumn{4}{|c|}{$\mathrm{D} 1$} & \multicolumn{4}{c}{$\mathrm{D} 26$} \\
\cline { 2 - 9 } & \multicolumn{3}{|c|}{ Age at the Beginning of Sickness } & \multicolumn{3}{c}{ Age at the Beginning of Sickness } \\
\cline { 2 - 8 } & 30 & 40 & 50 & 60 & 30 & 40 & 50 & 60 \\
\hline 2 & $\simeq 0$ & $\simeq 0$ & 0.0002 & 0.0076 & $\simeq 0$ & $\simeq 0$ & 0.0005 & 0.0229 \\
5 & 0.0267 & 0.06 & 0.1311 & 0.2825 & 0.1464 & 0.2471 & 0.407 & 0.6639 \\
10 & $\simeq 0$ & $\simeq 0$ & 0.0001 & 0.0021 & 0.0009 & 0.0092 & 0.066 & 0.3258 \\
15,18 & 0.0002 & 0.0016 & 0.0097 & 0.0525 & 0.0253 & 0.0802 & 0.2185 & 0.5204 \\
\hline \hline
\end{tabular}

After analysing all the results obtained, we have concluded that: for a given combination of sickness category and age at the beginning of sickness, the probability increases as the deferred period becomes longer; for a given combination of deferred period and sickness category, the probability increases as the age at the beginning of sickness becomes higher; and, in general, the probability is higher for sickness categories with the $\rho(i)_{x, z}$ having lower levels (in this last analysis we do not consider sickness category 2 (malignant neoplasms) which has an approximation to the $v(2)_{x, z}$ with a much higher level than the others). If we think carefully about these features, we can see that, intuitively, all of them were expected (recall that approximations to the $\rho(i)_{x, z}$ with lower levels mean longer claims).

We can also conclude that, depending on the values of $d, i$ and $y$, $65-y-d p_{y+\frac{d d d}{S_{i} S_{i}}}$ can be surprisingly high. See, for example, the values of $65-60-1 / 2 p_{60+1 / 2,1 / 2}$ and ${ }_{65-60-1 / 2} p_{60+1 / 2,1 / 2}$. The value of this probability can be significant even for early ages at the beginning of sickness and not very long deferred periods. Consider, for example, ${ }_{65-30-1 / 4} p_{30+1 / 4,1 / 4}=0.101$.

In the light of the information given in the previous paragraphs, we can conclude that, after all, the behaviour of the claim durations not conditioned on any mode of claim termination for high ages at the beginning of sickness, sickness categories with approximations to the $\rho(i)_{x, z}$ having low levels and long 
deferred periods was expected. The behaviour of the durations of claims which end in expiry, mentioned above, contributes to make the claims in Table 5, in general, much longer than those which end in recovery, specially for ages at the beginning of sickness above 40 (for the worst cases we have claims which are 8,9 and 10 times longer than those which end in recovery).

The other features we can observe in Table 5 were already observed in claim durations conditioned on recovery and in claim durations conditioned on death: in general, for a given combination of sickness category and age at the beginning of sickness, the average claim duration increases as the deferred period becomes longer and, for most of the combinations of deferred period and sickness category, the average claim duration increases up to some age at the beginning of sickness and then decreases. However, in this table: in general, the claim durations start to decrease at much later ages than those conditioned on recovery; we observe more cases where the average duration is an increasing function of $y$ than in the durations conditioned on recovery; and, unlike in the claims which end in recovery and in death, the average duration is a decreasing function of $y$ only for sickness category 2 and all the deferred periods we consider. It is obvious that the main reason for these differences is also the behaviour of the claim durations conditioned on expiry.

The results in Table 5 show that insurance companies should also analyse claim durations not conditioned on any mode of claim termination. In fact, considering that, when a claim is reported, the insurance company does not know in advance its mode of termination, and the durations in Table 5, in general, are much longer than those conditioned on recovery, to analyse only the latter average durations could be quite misleading for the persons who are controlling the durations of the claims which are being paid. Another conclusion we can draw, it is that claims which end in expiry are a phenomenon which should not be ignored by insurance companies. As we have seen above, these are the claims with the longest possible durations and their number becomes quite important for longer deferred periods, later ages at the beginning of sickness and sickness categories with approximations to the $\rho(i)_{x, z}$ having lower levels.

\section{ACKNOWLEDGEMENTS}

I am grateful to the associate editor responsible for this paper and three anonymous referees for their invaluable comments and suggestions.

\section{REFERENCES}

Committee of the SOA to Recommend New Disability Tables for Valuation (1985) Report of the Committee to Recommend New Disability Tables for Valuation. Transactions of Society of Actuaries 37, 449-601.

Continuous Mortality Investigation Committee (1991) The Analysis of Permanent Health Insurance Data. Continuous Mortality Investigation Reports 12. The Institute of Actuaries and the Faculty of Actuaries. 
Continuous Mortality Investigation Committee (1996) Recovery and Mortality Rates of Those Claiming Under PHI Policies, Individual 1975-90 and Group 1975-86. Continuous Mortality Investigation Reports 15. The Institute of Actuaries and the Faculty of Actuaries.

Continuous Mortality Investigation Committee (2000) Sickness Experience 1991-94 for Individual PHI Policies. Continuous Mortality Investigation Reports 18. The Institute of Actuaries and the Faculty of Actuaries.

Continuous Mortality Investigation Committee (2001) Sickness Experience 1995-98 for Individual Income Protection Policies. Continuous Mortality Investigation Reports 20. The Institute of Actuaries and the Faculty of Actuaries.

Continuous Mortality Investigation Committee (2005) Sickness Experience 1999-2002 for Individual Income Protection Policies. Continuous Mortality Investigation Reports 22. The Institute of Actuaries and the Faculty of Actuaries.

Cordeiro, I.M.F. (2002a) A Multiple State Model for the Analysis of Permanent Health Insurance Claims by Cause of Disability. Insurance: Mathematics and Economics 30(2), 167-186.

Cordeiro, I.M.F. (2002b) Transition Intensities for a Model for Permanent Health Insurance. Astin Bulletin 32(2), 319-346.

Haberman, S. and Pitacco, E. (1999) Actuarial Models for Disability Insurance. Chapman \& Hall/CRC, London.

Hoem, J.M. (1969) Some Notes on the Qualifying Period in Disability Insurance II. Problems of Maximum Likelihood Estimation. Mitteilungen der Vereinigung schweizerischer Versicherungsmathematiker 69, 301-317.

Hoem, J.M. (1972) Inhomogeneous Semi-Markov Processes, Select Actuarial Tables and Duration Dependence in Demography. In Population Dynamics (ed. T.N.E. Greville), Academic Press, New York.

Hoem, J.M. (1976) The Statistical Theory of Demographic Rates - A Review of Current Developments. Scandinavian Journal of Statistics 3, 169-185.

Hoem, J.M. (1988) The Versatility of the Markov Chain as a Tool in the Mathematics of Life Insurance. Transactions of the 23rd International Congress of Actuaries R, 171-202.

Income Protection Committee (2006) Analysis of Individual Income Protection Experience by Cause of Disability. Continuous Mortality Investigation Working Paper 23. The Institute of Actuaries and the Faculty of Actuaries.

Individual Disability EXPERIENCe CommitTeE (2005) 1990-99 Individual Disability Experience Committee Report. Society of Actuaries.

Waters, H.R. (1984) An Approach to the Study of Multiple State Models. The Journal of the Institute of Actuaries Part II: 111(448), 363-374.

Waters, H.R. (1990) The Recursive Calculation of the Moments of the Profit on a Sickness Insurance Policy. Insurance: Mathematics and Economics 9, 101-113.

Wolthuis, H. (1994) Life Insurance Mathematics (The Markovian Model). CAIRE Education Series, Vol. 2.

\author{
IsAbel Maria Ferraz Cordeiro \\ Escola de Economia e Gestão \\ Universidade do Minho \\ Campus Universitário de Gualtar \\ 4710-057 Braga \\ Portugal \\ Tel: $++351-253-604563$ \\ Fax: ++351-253-676375 \\ E-mail: icordeiro@eeg.uminho.pt
}

